\title{
Fatores psicológicos, bruxismo e atividade física em adolescentes: Revisão integrativa
}

\author{
Psychological factors, bruxism and physical activity in adolescents: An integrative review \\ Factores psicológicos, bruxismo y actividad física en adolescentes: Revisión integradora
}

Recebido: 02/02/2022 | Revisado: 06/02/2022 | Aceito: 13/02/2022 | Publicado: 19/02/2022

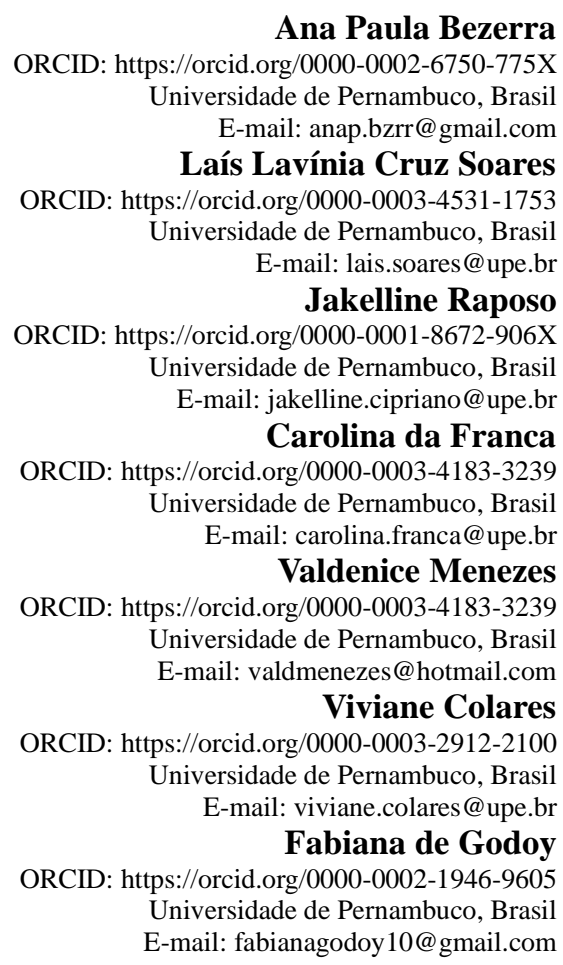

\section{Resumo}

Esta revisão integrativa teve o objetivo de analisar a relação entre o bruxismo, a atividade física e os fatores psicológicos nos adolescentes. O levantamento bibliográfico teve início em fevereiro de 2020 e foi finalizado em agosto de 2021, através do acesso online a quatro bases de dados: Pubmed (National Library of Medicine), LILACS Literatura Latino Americana e do Caribe em Ciências da Saúde e Scielo (Scientific Electronic Library Online), a fim de responder a seguinte pergunta condutora: É possível estabelecer uma relação entre os fatores psicológicos associados ao bruxismo e a atividade física? Foram utilizados um conjunto de descritores em português e seus correspondentes em inglês. Devido aos poucos estudos sobre tema, foram realizadas duas buscas separadas, uma com foco no bruxismo e outra com foco na atividade física. Os artigos selecionados foram publicados de 2010 a 2021 . O bruxismo do sono foi mais prevalente na população de adolescentes, assim como o sexo feminino nos dois tipos (bruxismo de vigília e bruxismo do sono). Na atividade física, os meninos foram mais ativos que as meninas. A ansiedade foi mais frequente entre os achados, tanto no bruxismo, como na atividade física. Não foi possível estabelecer uma associação entre bruxismo, atividade física e fatores psicológicos, contudo esses fatores estão associados com ambos.

Palavras-chave: Exercício físico; Medicina do comportamento; Bruxismo; Adolescente.

\begin{abstract}
This integrative review aimed to analyze the relationship between bruxism, physical activity, and psychological factors in adolescents. The bibliographical survey was started in February 2020 and completed in August 2021, through online access to four databases: Pubmed (National Library of Medicine), LILACS - Latin American and Caribbean Literature on Health Sciences and Scielo (Scientific Electronic Library Online), in to answer the following research question: Is it possible to establish a relationship between psychological factors associated with bruxism and physical activity? A set of descriptors in Portuguese and their counterparts in English were used. Due to the few studies on the subject, two separate searches were carried out, one focusing on bruxism and the other focusing on physical activity. The selected articles were published from 2010 to 2021. Sleep bruxism was more prevalent in the adolescent population, as was female in both types (wake bruxism and sleep bruxism). In physical activity, boys were
\end{abstract}


more active than girls. Anxiety was more frequent among the findings, both in bruxism and physical activity. It was not possible to establish an association between bruxism, physical activity, and psychological factors, but these factors are associated with both.

Keywords: Physical exercise; Behavioral medicine; Bruxism; Adolescent.

\section{Resumen}

Esta revisión integradora tuvo como objetivo analizar la relación entre bruxismo, actividad física y factores psicológicos en adolescentes. La encuesta bibliográfica comenzó en febrero de 2020 y se completó en agosto de 2021, mediante el acceso en línea a cuatro bases de datos: Pubmed (Biblioteca Nacional de Medicina), LILACS - Literatura Latinoamericana y del Caribe en Ciencias de la Salud y Scielo (Biblioteca Electrónica Científica en Línea), para dar respuesta a la siguiente pregunta principal: ¿Es posible establecer una relación entre los factores psicológicos asociados al bruxismo y la actividad física? Se utilizó un conjunto de descriptores en portugués y sus contrapartes en inglés. Debido a los pocos estudios sobre el tema, se llevaron a cabo dos búsquedas separadas, una centrada en el bruxismo y la otra centrada en la actividad física. Los artículos seleccionados fueron publicados entre 2010 y 2021 . El bruxismo del sueño fue más prevalente en la población adolescente, al igual que el femenino en ambos tipos (bruxismo de vigilia y bruxismo del sueño). En la actividad física, los niños eran más activos que las niñas. La ansiedad fue más frecuente entre los hallazgos, tanto en el bruxismo como en la actividad física. No fue posible establecer una asociación entre bruxismo, actividad física y factores psicológicos, sin embargo, estos factores se asocian con ambos.

Palabras clave: Ejercicio físico; Medicina de la conducta; Bruxismo; Adolescente.

\section{Introdução}

A adolescência é a fase que acompanha mudanças constantes em hábitos e formas de enxergar o mundo. Mundo esse, que também está em transformação, especificamente em relação aos comportamentos que competem com a prática regular de atividade física (Lírio, 2012). Tais estímulos são ainda mais reforçados entre os adolescentes e estão voltados para os comportamentos e potencialização de estilos de vida sedentários. Tal assunto deve ser avaliado quanto à sua relevância no desenvolvimento do bruxismo, uma vez que todos se relacionam com os fatores psicológicos (Alves, 2021).

A prática de atividade física se refere a qualquer tipo de movimento corporal, isso inclui: caminhada, jogos, ciclismo, recreação e pode ser feito em qualquer nível de habilidade em busca do prazer. Recomendada pela WHO, crianças e adolescentes devem fazer em média 60 minutos por dia, de intensidade moderada a vigorosa, para resultados satisfatórios na saúde. (World Health Organization, 2020). Já o bruxismo, considerado como um comportamento motor, caracterizado pelo apertamento ou ranger dos dentes, é uma condição que pode ocorrer durante o sono ou no estado de vigília e pode acarretar em alterações craniofaciais e temporomandibulares, além de desgastes dentários, fadiga acompanhada de dores e prejuízos musculares. Somando-se a isso, o acúmulo de estresse e ansiedade acentuados diante da rotina agitada e das novas responsabilidades da futura vida adulta; além do abandono de práticas saudáveis, alegado pela falta de tempo ou substituídas por práticas ociosas, estimulam o comportamento sedentário e/ou a inatividade física; podendo provocar comportamentos orais como o bruxismo, interferindo diretamente na qualidade de vida do adolescente (Antonio, et al., 2006; Alencar, et al, 2020; Alves, 2021).

O presente estudo de revisão integrativa da literatura teve o objetivo de investigar a relação entre fatores psicológicos, bruxismo e atividade física na população de adolescentes de 10 a 19 anos de idade.

\section{Metodologia}

Trata-se de uma revisão integrativa da literatura que pretende investigar a relação do bruxismo e da prática de atividade física entre os adolescentes, através de um método de pesquisa minucioso e soma de fontes do determinado assunto, de forma sistemática, objetiva e abrangente, com o objetivo de responder a seguinte pergunta condutora: "É possível estabelecer uma relação entre os fatores psicológicos associados ao bruxismo e a atividade física?”. 
Este trabalho foi realizado em três estágios, de acordo com a metodologia da revisão integrativa. Primeiro, o levantamento bibliográfico foi feito nas bases de dados: BVS/ Portal Bireme (Medline - Literatura Internacional em Ciências da Saúde), LILACS - Literatura Latino Americana e do Caribe em Ciências da Saúde e Pubmed (National Library of Medicine - NIH). Em seguida, foi feita a seleção dos trabalhos que abordavam os temas: "atividade física" e/ou "bruxismo" e/ou fatores psicológicos e cuja formulação fosse igual ou semelhante às propostas na busca realizada para o presente estudo; realizou-se a leitura dos títulos e resumos dos artigos previamente selecionados. Porém, antes de iniciar a leitura dos resumos, os títulos foram identificados e aqueles em duplicidade, ou seja, aparecendo em mais de uma base de dados foram retirados da análise. $\mathrm{Na}$ terceira e última etapa constituiu-se da leitura dos textos na íntegra, seguida pela construção de uma tabela com as informações levantadas nesse processo.

Para inclusão dos artigos foram considerados os seguintes critérios de seleção: artigos que abordassem o assunto sobre a prática de atividade física, artigos que contemplassem o tema bruxismo e os fatores psicológicos associados com os dois assuntos. Também, estudos cuja população-alvo fosse formada por adolescentes na faixa etária de 10 a 19 anos. Os estudos selecionados datavam do ano de 2010 a 2021, não houve limitação de idioma. Os critérios de exclusão envolveram todo tipo de estudo de revisão da literatura e que não apresentassem resultados para a faixa etária da adolescência de forma isolada.

O levantamento bibliográfico iniciou-se em fevereiro de 2020 até agosto de 2021, através do acesso online a três bases de dados: Pubmed (National Library of Medicine), LILACS - Literatura Latino Americana e do Caribe em Ciências da Saúde e Scielo (Scientific Electronic Library Online). Foram utilizados um conjunto de descritores em português e seus correspondentes em inglês para que fossem incluídos o maior número de pesquisas referentes ao tema. Devido aos poucos estudos sobre tema, foram realizadas duas buscas separadas, uma com foco no bruxismo, com os descritores: "bruxismo", "saúde mental", "adolescente"; "bruxismo", "ansiedade", "adolescente”; "bruxismo", "estresse”, "adolescente”, "bruxismo", "depressão", "adolescente”; e seus correspondentes em inglês: "bruxism”, "mental health”, "adolescent”; "bruxism”, “anxiety", "adolescent”; "bruxism", "stress", “adolescent”, "bruxism", “depression”, “adolescent”. A outra busca, relacionada à atividade física, teve como termos: "atividade física", "saúde mental", "adolescente"; "atividade física", "ansiedade", "adolescente"; "atividade física", "estresse", "adolescente", "atividade física", "depressão", "adolescente"; e em inglês: "physical activity", "mental health" and "adolescent"; "physical activity", "anxiety", "adolescent"; "physical activity", "stress", "adolescent", "physical activity", "depression", "adolescent". Foi usado o operador booleano AND, fornecendo intercessão na busca. Os fluxogramas das duas buscas estão apresentados nas figuras 1 e 2. 
Research, Society and Development, v. 11, n. 3, e22811326450, 2022

(CC BY 4.0) | ISSN 2525-3409 | DOI: http://dx.doi.org/10.33448/rsd-v11i3.26450

Figura 1 - Fluxograma da busca sobre bruxismo e saúde mental.
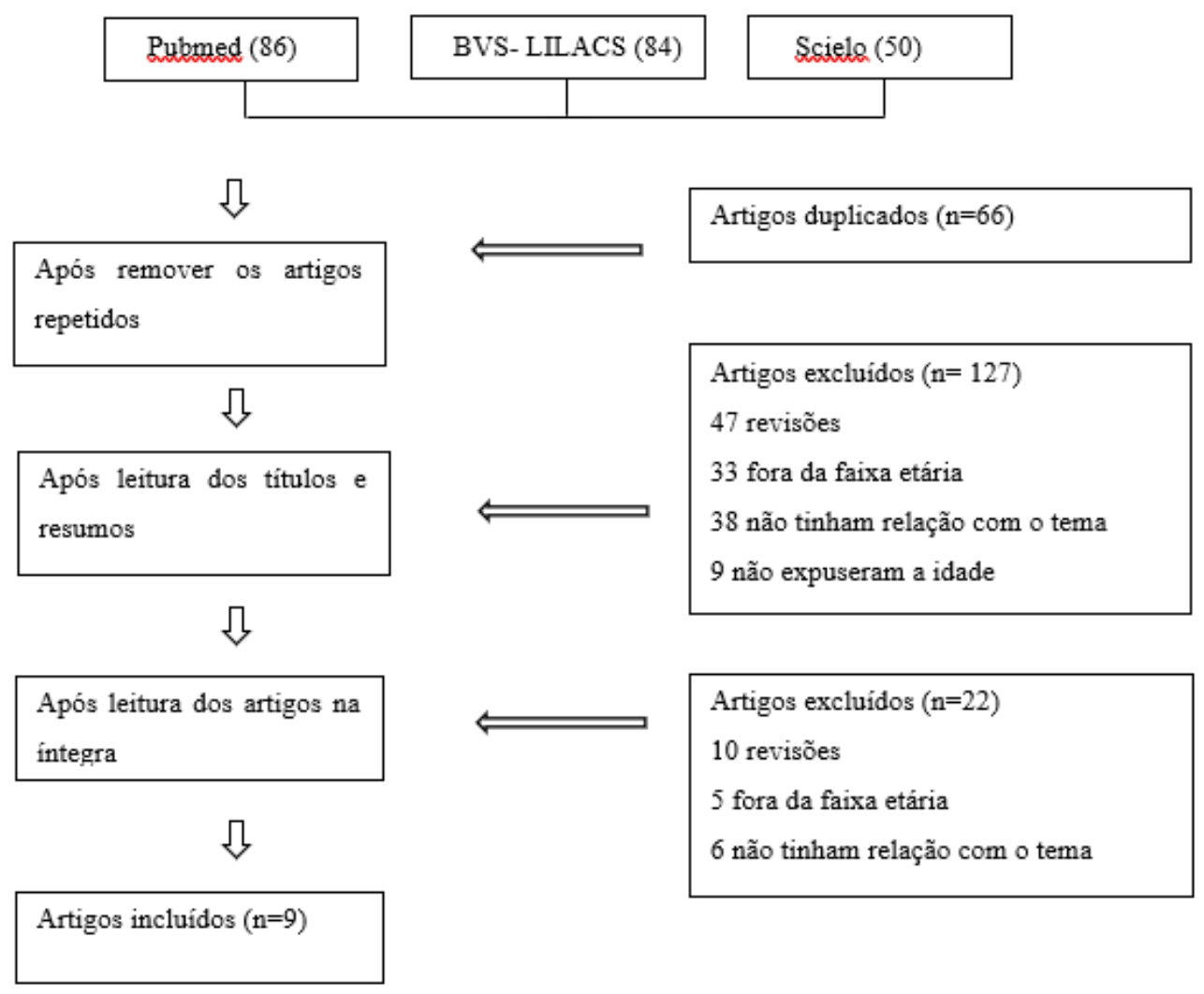

Fonte: Bezerra, et al. (2022).

A Figura 1 corresponde ao fluxograma da $1^{\text {a }}$ busca, com foco no bruxismo e fatores psicológicos, tais como depressão, ansiedade e estresse. 
Figura 2 - Fluxograma da busca sobre atividade física e saúde mental.

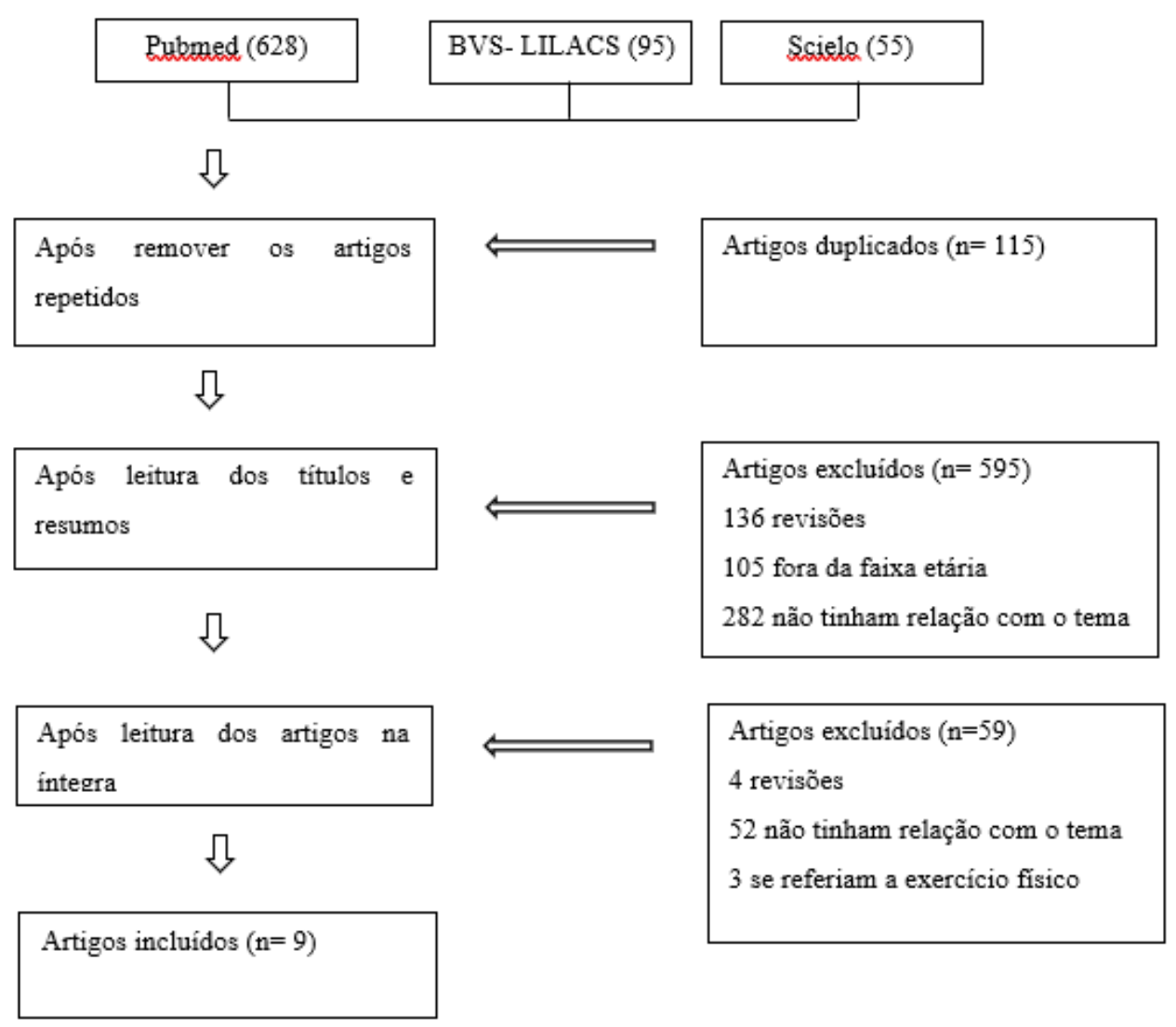

Fonte: Bezerra, et al. (2022).

A Figura 2 corresponde ao fluxograma da $2^{\mathrm{a}}$ busca, com foco na atividade física e fatores psicológicos, tais como depressão, ansiedade e estresse.

\section{Resultados}

Após a aplicação da estratégia de busca e dos critérios de elegibilidade, foram selecionados 19 artigos, que compuseram o presente estudo. Dentre os trabalhos selecionados, todos dispuseram de desenho metodológico de corte transversal. Nota-se que dentre os dezenove estudos, existe uma diversidade de países, sendo: oito no Brasil, dois na Holanda, dois em Israel, dois na Espanha e um em cada país diferente: Lituânia, Turquia, Cuba, Austrália, México. Referente a amostra, a maioria dos artigos coletaram dados de adolescentes de ambos os sexos.

Em relação aos estudos do bruxismo, a maioria dos trabalhos abordaram a faixa etária entre 10 e 19 anos, somando-se todos os estudos tem-se um total de 9.475 adolescentes pesquisados. Também, a maioria se baseou em questionários de autorrelato, três acrescentaram o exame clínico à avaliação. Apenas um estudo não aplicou questionários de autorrelato com adolescentes ou responsáveis (Tabela 1).

Nem todos analisaram os dois tipos de bruxismo (sono e vigília). Quatro artigos abordaram apenas sobre o bruxismo do sono e seis sobre ambos. Dos 10 estudos, quatro observaram a prevalência do sexo feminino no desencadeamento do bruxismo. E a idade mais afetada foi variada, quatro não informaram e apenas em um não houve diferença estatisticamente significativa. Os fatores psicológicos associados mais frequentes foram estresse e ansiedade, apenas dois artigos não tinham objetivo de analisar os fatores mentais (Tabela 2). 
Tabela 1 - Distribuição dos estudos sobre bruxismo de acordo com país, autor, ano, desenho do estudo, amostra, idade e instrumento diagnóstico.

\begin{tabular}{|c|c|c|c|c|c|}
\hline País & Autor/ano & Desenho do estudo & Amostra & Idade & Instrumento diagnóstico \\
\hline \multirow[t]{2}{*}{ Holanda } & Selms, et al., (2012) & Transversal & 4.235 adolescentes & 12 a 18 anos & $\begin{array}{l}\text { Questionário de autorrelato (Research } \\
\text { Diagnostic Criteria for Temporomandibular } \\
\text { Disorders) e questionário de parafunções com } \\
\text { adolescentes }\end{array}$ \\
\hline & $\begin{array}{l}\text { Wetselaar, et al., } \\
(2021)\end{array}$ & Transversal & 920 adolescentes & 17 anos & Questionário de autorrelato com adolescentes \\
\hline Turquia & $\begin{array}{l}\text { Türkoğlu, et al., } \\
(2013)\end{array}$ & Transversal & 70 adolescentes & 8 a 17 anos & Questionário de autorrelato com adolescentes \\
\hline \multirow[t]{3}{*}{ Brasil } & $\begin{array}{l}\text { Carvalho, et al., } \\
(2015)\end{array}$ & Transversal & 594 adolescentes & 11 a 14 anos & $\begin{array}{l}\text { Relatos de responsáveis legais e a qualidade de } \\
\text { vida foi avaliada através da versão brasileira do } \\
\text { questionário CPQ11-14, na forma curta }\end{array}$ \\
\hline & Sousa, et al., (2018) & Transversal & 594 adolescentes & 11 a 14 anos & $\begin{array}{l}\text { Questionário com pais e responsáveis e exame } \\
\text { clínico }\end{array}$ \\
\hline & Prado, et al., (2018) & Transversal & 239 adolescentes & 12 anos & $\begin{array}{l}\text { Questionário de autorrelato com adolescentes, } \\
\text { responsáveis legais e exame clínico extra e } \\
\text { intraoral }\end{array}$ \\
\hline Israel & Winocur, et al., (2019) & Transversal & 2.347 adolescentes & $\begin{array}{l}\text { Idade média de } 15,7 \text { anos } \\
\text { (desvio-padrão de } 1,1 \text { anos) }\end{array}$ & Questionário de autorrelato on-line \\
\hline Cuba & Ayala \& Llana (2019) & Transversal & 198 adolescentes & 16 a 18 anos & Prontuários médicos individuais \\
\hline Espanha & $\begin{array}{l}\text { Carrillo-Diaz, et al., } \\
(2021)\end{array}$ & Transversal & 213 adolescentes & $11-17$ anos & Questionário de autorrelato com adolescentes \\
\hline México & Murrieta, et al., (2014) & Transversal & 278 adolescentes & 16 a 18 anos & $\begin{array}{l}\text { Questionário de autorrelato com adolescentes e } \\
\text { exame oral }\end{array}$ \\
\hline
\end{tabular}

Fonte: Bezerra, et al. (2022). 


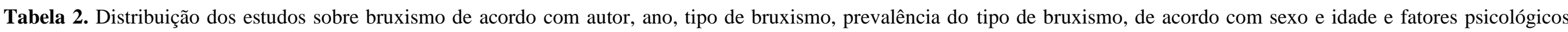
associados.

\begin{tabular}{|c|c|c|c|c|c|}
\hline Autor/ano & $\begin{array}{l}\text { Tipo de } \\
\text { bruxismo }\end{array}$ & $\begin{array}{l}\text { Prevalência } \\
\text { do tipo de } \\
\text { bruxismo }\end{array}$ & $\begin{array}{l}\text { Prevalência de acordo com o } \\
\text { sexo }\end{array}$ & Prevalência de acordo com a idade & $\begin{array}{ll}\text { Fatores } & \text { psicológicos } \\
\text { associados } & \end{array}$ \\
\hline $\begin{array}{l}\text { Selms, et al., } \\
\text { (2012) }\end{array}$ & Ambos & $\begin{array}{l}\text { BS: } 14,8 \%, \\
\text { BV: } 8,7 \%\end{array}$ & $\begin{array}{l}\text { BS: Sexo feminino: } 49,9 \% \text {, sexo } \\
\text { masculino: } 32,9 \% \\
\text { BV: Sexo feminino: } 23,14 \% \text {, sexo } \\
\text { masculino no BV: } 52 \%\end{array}$ & $\begin{array}{l}\text { BS: Sexo feminino: } 12 \text { anos }(37 \%), 13 \text { anos }(75 \%), 14 \text { anos }(61 \%), 15 \text { anos } \\
(73 \%), 16 \text { anos }(60 \%), 17 \text { anos }(32 \%), 18 \text { anos }(11 \%) \text {. Sexo masculino: } 12 \text { anos } \\
(23 \%), 13 \text { anos }(47 \%), 14 \text { anos }(33 \%), 15 \text { anos }(56 \%), 16 \text { anos }(35 \%), 17 \text { anos } \\
(31 \%), 18 \text { anos }(5 \%) \\
\text { BV: Sexo feminino: } 12 \text { anos }(13 \%), 13 \text { anos }(25 \%), 14 \text { anos }(31 \%), 15 \text { anos } \\
(39 \%), 16 \text { anos }(31 \%), 17 \text { anos }(18 \%), 18 \text { anos }(5 \%) . \text { Sexo masculino: } 12 \text { anos } \\
(15 \%), 13 \text { anos }(32 \%), 14 \text { anos }(27 \%), 15 \text { anos }(34 \%), 16 \text { anos }(34 \%), 17 \text { anos } \\
(34 \%), 18 \text { anos }(6 \%)\end{array}$ & $\begin{array}{l}\text { Estresse, } \\
\text { depressivo }\end{array}$ \\
\hline $\begin{array}{l}\text { Wetselaar, et } \\
\text { al., (2021) }\end{array}$ & Ambos & $\begin{array}{l}\text { BS: } 7,6 \%, \mathrm{BV}: \\
4,1 \%\end{array}$ & $\begin{array}{l}\text { BS: Sexo feminino: } 7,8 \% \text {, sexo } \\
\text { masculino: } 7,5 \% \\
\text { BV: Sexo feminino: } 5 \% \text {, sexo } \\
\text { masculino: } 3,2 \%\end{array}$ & $\begin{array}{l}\text { BS: } 17 \text { anos: Sexo feminino: 7,8\%, sexo masculino: 7,5\%. } \\
\text { BV: } 17 \text { anos: Sexo feminino: 5\%, sexo masculino: } 3,2 \% \text {. }\end{array}$ & Ansiedade odontológica \\
\hline $\begin{array}{l}\text { Türkoğlu, et } \\
\text { al., (2013) }\end{array}$ & $\begin{array}{l}\text { Bruxismo } \\
\text { do sono }\end{array}$ & NI & NI & $\mathrm{NI}$ & Ansiedade, depressão \\
\hline $\begin{array}{l}\text { Carvalho, et } \\
\text { al., (2015) }\end{array}$ & $\begin{array}{l}\text { Bruxismo } \\
\text { do sono }\end{array}$ & BS: $22,2 \%$ & $\begin{array}{l}\text { BS: Sexo feminino: } 53,8 \% \text {, sexo } \\
\text { masculino: } 46,2 \%\end{array}$ & $11 \operatorname{anos}(28 \%), 12$ anos $(31,1 \%), 13$ anos $(13,6 \%), 14$ anos $(27,3 \%)$ & $\begin{array}{l}\text { Qualidade de vida (bem } \\
\text { estar social e emocional) }\end{array}$ \\
\hline $\begin{array}{l}\text { Sousa, et al., } \\
(\mathbf{2 0 1 8 )}\end{array}$ & $\begin{array}{l}\text { Bruxismo } \\
\text { do sono }\end{array}$ & BS: $22,2 \%$ & $\begin{array}{l}\text { BS: Sexo feminino: } 18,9 \% \text {, sexo } \\
\text { masculino: } 27,9 \%\end{array}$ & $11 \operatorname{anos}(24 \%), 12$ anos $(25,5 \%), 13$ anos $(13 \%), 14$ anos $(25,5 \%)$ & Ansiedade \\
\hline $\begin{array}{l}\text { Prado, et al., } \\
(2018)\end{array}$ & $\begin{array}{l}\text { Bruxismo } \\
\text { do sono }\end{array}$ & $\begin{array}{l}\text { BS: } 16,9 \%, \\
\text { BV: } 23,9 \%\end{array}$ & $\begin{array}{l}\text { BS: Sexo feminino: } 53,8 \% \text {, sexo } \\
\text { masculino: } 46,2 \%\end{array}$ & $\mathrm{NI}$ & Nenhum \\
\hline $\begin{array}{l}\text { Winocur, et } \\
\text { al., (2019) }\end{array}$ & Ambos & $\begin{array}{l}\text { BS: } 14,8 \%, \\
\text { BV: } 34,5 \% \text { e } \\
7,3 \% \text { ambos }\end{array}$ & $\begin{array}{l}\text { BS: Sexo feminino: } 24,1 \% \text {, sexo } \\
\text { masculino: } 15,4 \% \\
\text { BV: Sexo feminino: } 32,1 \% \text {, sexo } \\
\text { masculino: } 30,1 \%\end{array}$ & $\begin{array}{l}\text { BS: } 14 \text { anos: } 19,4 \%, 15 \text { anos: } 19,3 \%, 16 \text { anos: } 21,8 \%, 17 \text { anos: } 19,2 \%, 18 \text { anos: } \\
\text { 15,7\% } \\
\text { BV: } 14 \text { anos:29,2\%, } 15 \text { anos: } 33,8 \%, 16 \text { anos: } 35 \%, 17 \text { anos: } 27,6 \%, 18 \text { anos: } \\
22 \%\end{array}$ & Ansiedade, estresse \\
\hline $\begin{array}{l}\text { Ayala \& Llana } \\
(\mathbf{2 0 1 9 )}\end{array}$ & Ambos & NI & $\begin{array}{l}\text { Sexo feminino: } 53,5 \%, \text { sexo } \\
\text { masculino: } 46,4 \%\end{array}$ & $\begin{array}{l}\text { Sexo feminino: } 16 \text { anos: } 34 \%, 17 \text { anos: } 27,4 \%, 18 \text { anos: } 38,7 \% \text {, sexo masculino: } \\
16 \text { anos: } 33,7 \% 18,17 \text { anos: } 28,3 \%, 18 \text { anos }(38 \%)\end{array}$ & $\begin{array}{l}\text { Estresse, ansiedade } \\
\text { transtornos do sono }\end{array}$ \\
\hline $\begin{array}{l}\text { Carrillo-Diaz, } \\
\text { et al., (2021) }\end{array}$ & Ambos & NI & NI & NI & Ansiedade \\
\hline $\begin{array}{l}\text { Murrieta, et } \\
\text { al., (2014) }\end{array}$ & Ambos & $\begin{array}{l}47,5 \% \quad \text { dos } \\
\text { adolescentes } * *\end{array}$ & $\begin{array}{l}\text { Não houve diferença } \\
\text { estatisticamente significativa }\end{array}$ & $\begin{array}{l}\text { Sexo masculino: } 16 \text { anos: } 11,5 \%, 17 \text { anos: } 30,6 \%, 18 \text { anos: } 8,6 \% \text {, sexo feminino: } \\
16 \text { anos: } 19,8 \%, 17 \text { anos: } 63,3 \%, 18 \text { anos: } 16,9 \%\end{array}$ & Nenhum \\
\hline
\end{tabular}

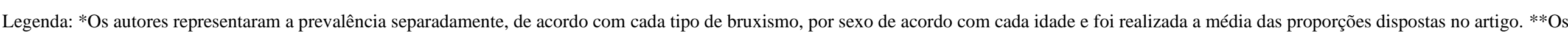
autores não diferenciam a prevalência de acordo com os tipos de bruxismo. Fonte: Bezerra, et al. (2022). 
Sobre os estudos da prática de atividade física, a maioria foi realizada no Brasil. Envolveram a faixa etária da adolescência (10 aos 19 anos), apenas um trabalho envolveu dos

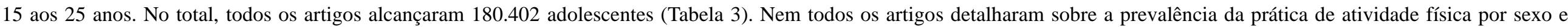
idade.

Dos nove artigos selecionados, cinco relataram que o sexo masculino foi o mais ativo fisicamente, três não informaram e um usou uma amostra de escolares apenas do sexo

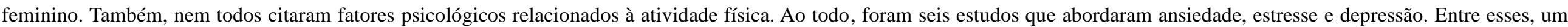
observou a qualidade de vida dos adolescentes e outro, o aspecto do bem-estar (Tabela 4).

Tabela 3 - Distribuição dos estudos sobre atividade física de acordo com país, autor, ano, desenho do estudo, amostra, idade e instrumento de avaliação.

\begin{tabular}{|c|c|c|c|c|c|}
\hline País & Autor/ano & $\begin{array}{ll}\begin{array}{l}\text { Desenho } \\
\text { estudo }\end{array} & \text { do } \\
\end{array}$ & Amostra & Idade & Instrumento de avaliação \\
\hline Austrália & $\begin{array}{l}\text { Parker, et al., } \\
(2016)\end{array}$ & Transversal & $\begin{array}{l}160 \\
\text { adolescentes }\end{array}$ & 15 a 25 anos & $\begin{array}{l}\text { Sessões de intervenção: 1: atividade física de ativação comportamental e } \\
\text { terapia de resolução de problemas (PST); 2: atividade física e de suporte } \\
\text { aconselhamento; 3: Psicoeducação e PST; e 4: Psicoeducação e } \\
\text { aconselhamento de apoio }\end{array}$ \\
\hline \multirow[t]{5}{*}{ Brasil } & $\begin{array}{l}\text { Navarro, et al., } \\
(2018)\end{array}$ & Transversal & $\begin{array}{l}200 \\
\text { adolescentes }\end{array}$ & 10 a 19 anos & Questionário de autorrelato \\
\hline & $\begin{array}{l}\text { Santana, et al., } \\
(2021)\end{array}$ & Transversal & $\begin{array}{l}102.072 \\
\text { adolescentes }\end{array}$ & 11 a 16 anos & Questionários de autorrelato em smartphones \\
\hline & $\begin{array}{l}\text { Ferreira, et al., } \\
(2020)\end{array}$ & Transversal & $\begin{array}{l}73.399 \\
\text { adolescentes }\end{array}$ & 12 a 17 anos & $\begin{array}{l}\text { Questionários de autorrelato e para avaliar o nível de atividade física foi usado } \\
\text { uma adaptação do self-administered physical activity checklist }\end{array}$ \\
\hline & $\begin{array}{lll}\text { Freire, et al., } \\
(2014)\end{array}$ & Transversal & $\begin{array}{l}763 \\
\text { adolescentes }\end{array}$ & 15 a 19 anos & Questionários em visitas domiciliares \\
\hline & $\begin{array}{l}\text { Bacil, et al., } \\
(2020)\end{array}$ & Transversal & $\begin{array}{l}2.347 \\
\text { adolescentes }\end{array}$ & 11 a 15 anos & Questionários de autorrelato, medição antropométrica e de maturação sexual. \\
\hline Lituânia & $\begin{array}{l}\text { Malinauskas \& } \\
\text { Malinauskaiene, } \\
(2015)\end{array}$ & Transversal & $\begin{array}{l}1.015 \\
\text { adolescentes }\end{array}$ & 14 a 15 anos & Questionários de autorrelato \\
\hline Espanha & $\begin{array}{l}\text { Carrillo-Diaz, et } \\
\text { al., (2021) }\end{array}$ & Transversal & $\begin{array}{l}213 \\
\text { adolescentes }\end{array}$ & 11 a 17 anos & Questionários de autorrelato e exame clínico \\
\hline Israel & $\begin{array}{l}\text { Shennar-Golan \& } \\
\text { Walter (2018) }\end{array}$ & Transversal & $\begin{array}{l}233 \\
\text { adolescentes }\end{array}$ & 13 a 18 anos & Questionários de autorrelato \\
\hline
\end{tabular}

Fonte: Bezerra, et al. (2022). 


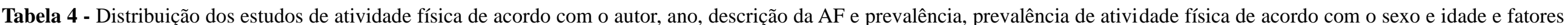
psicológicos associados.

\begin{tabular}{|c|c|c|c|c|}
\hline Autor/ano & Descrição da AF e prevalência & Prevalência de PAF de acordo com o sexo & $\begin{array}{l}\text { Prevalência de PAF de acordo } \\
\text { com a idade }\end{array}$ & $\begin{array}{l}\text { Fatores psicológicos } \\
\text { associados }\end{array}$ \\
\hline $\begin{array}{l}\text { Parker, et al., } \\
(2016)\end{array}$ & $\begin{array}{l}\text { Atividade: NI, inatividade: NI e } \\
\text { sedentarismo: NI }\end{array}$ & NI & NI & Depressão, ansiedade \\
\hline $\begin{array}{l}\text { Navarro, et al., } \\
(2018)\end{array}$ & $\begin{array}{l}\text { Atividade: NI, inatividade: NI e } \\
\text { sedentarismo: NI }\end{array}$ & $\begin{array}{l}\text { Meninos se apresentaram mais ativos do que } \\
\text { meninas. } 53,9 \% \text { do sexo masculino se exercitou } \\
\text { por 5/ou mais semanas, } 28,9 \% \text { por mais } \\
\text { 300min/semana e } 35,5 \% \text { ficou } 6 / \text { ou mais dias } \\
\text { sedentário, comparado com o sexo feminino, que } \\
\text { obteve os valores: } 35,4 \%, 13,7 \%, 42,7 \% \text {, } \\
\text { respectivamente. * }\end{array}$ & $\mathrm{NI}$ & Nenhum \\
\hline $\begin{array}{l}\text { Santana, et al., } \\
(2021)\end{array}$ & $\begin{array}{l}\text { Atividade: NI, inatividade: } 78,1 \% \\
\text { e sedentarismo: } 56,3 \%\end{array}$ & $\begin{array}{l}\text { Sexo masculino: mais ativo, com } 70,6 \% \text { de } \\
\text { inativos e } 54,8 \% \text { de sedentários. O sexo feminino } \\
\text { se apresentou mais inativo }(85,2 \%) \text { e sedentário } \\
(57,8 \%)\end{array}$ & $\begin{array}{l}\text { Inativos: } 10 \text { a } 13 \text { anos: } 80,3 \% \text {, } \\
14 \text { anos: } 78,7 \%, 15 \text { anos: } \\
75,5 \%, \geq 16 \text { anos: } 75,9 \% \\
\text { sedentários: } 10 \text { a } 13 \text { anos: } \\
59,2 \%, \quad 14 \text { anos: } 58,6 \%, 15 \\
\text { anos: } 52,8 \%, \geq 16 \text { anos: } 47,2 \%\end{array}$ & Nenhum \\
\hline $\begin{array}{l}\text { Ferreira, et al., } \\
(\mathbf{2 0 2 0})\end{array}$ & $\begin{array}{l}\text { Atividade: } 44,4 \%, \\
\text { insuficientemente ativos: } 26,5 \% \mathrm{e} \\
\text { inativos: } 29,1 \%\end{array}$ & NI & NI & $\begin{array}{lr}\text { Transtornos } & \text { mentais } \\
\text { comuns } & \text { (ansiedade, } \\
\text { depressão) } & \\
\end{array}$ \\
\hline $\begin{array}{lll}\text { Freire, } \\
(\mathbf{2 0 1 4})\end{array}$ et al., & Atividade: $45,2 \%$, inatividade: $\mathrm{NI}$ & $\begin{array}{l}\text { Ativos: Sexo masculino: } \mathrm{RP}=2,22 \text {, sexo feminino: } \\
\mathrm{RP}=1\end{array}$ & $\begin{array}{l}\text { Adolescentes mais jovens eram } \\
\text { mais ativos }(\mathrm{RP}=0,94)\end{array}$ & Qualidade de vida \\
\hline Bacil, et al., (2020) & $\begin{array}{l}\text { Atividade: } 52,3 \% \text {, inatividade: NI } \\
\text { e sedentarismo: NI }\end{array}$ & $\begin{array}{l}\text { Sexo masculino mais ativo: } 64,1 \% \text {, a prevalência } \\
\text { do sexo feminino não foi informada }\end{array}$ & $\mathrm{NI}$ & Nenhum \\
\hline $\begin{array}{l}\text { Malinauskas \& } \\
\text { Malinauskaiene } \\
(\mathbf{2 0 1 5})\end{array}$ & $\begin{array}{l}\text { Atividade: NI, inatividade: } 12,2 \% \\
\text { e sedentarismo: NI }\end{array}$ & $\begin{array}{l}\mathrm{O} \text { estudo foi feito com amostra de adolescentes } \\
\text { apenas do sexo feminino, em que } 12,2 \% \text { eram } \\
\text { inativas }\end{array}$ & $\mathrm{NI}$ & ansiedade, \\
\hline Diaz, et al., (2021) & $\begin{array}{l}\text { Atividade: NI, inatividade: NI e } \\
\text { sedentarismo: NI }\end{array}$ & NI & $\mathrm{NI}$ & Ansiedade \\
\hline $\begin{array}{l}\text { Shennar-Golan \& } \\
\text { Walter (2018) }\end{array}$ & $\begin{array}{l}\text { Atividade: NI, inatividade: NI e } \\
\text { sedentarismo: NI }\end{array}$ & $\begin{array}{l}\text { AF extenuante média foi maior para meninos }(\mathrm{M} \\
=3,9) \text { do que para meninas }(\mathrm{M}=1,8)\end{array}$ & $\mathrm{NI}$ & Bem estar \\
\hline
\end{tabular}

Legenda: * O autor não informa a prevalência, mas se baseia nos dias de exercícios/semana, minutos de exercício/semana e horas de sedentarismo/dia para avaliar o sexo em relação à prática de atividade física. NI: Não informado. Fonte: Bezerra, et al. (2022) 


\section{Discussão}

A prevalência do bruxismo do sono variou de $7,6 \%$ a $22,2 \%$. Já o de vigília, as taxas variaram em torno de $4,1 \%$ a 23,9\%. Para ambos os tipos, as prevalências foram de 7,3\% e 47,5\%. Wetselaar, et al., (2021) demonstraram que o BS foi mais comum do que o BV na população de adolescentes holandeses. Detectaram a prevalência de 7,6\% e 4,1\% respectivamente. Corroborando com Selms, et al., (2012) que demonstraram que a taxa de prevalência do BS e BV foi de $14,8 \%$ e $8,7 \%$, respectivamente.

Winocur, et al., (2019) concordam com Selms, et al., (2012) sobre a prevalência de 14,8\% para o bruxismo do sono. Porém, seu valor em relação ao bruxismo de vigília difere muito, com 34,5\%. Segundo Winocur, et al., (2019) essa frequência maior do BV se deve aos métodos que podem ter levado a diferenças nos relatos de hábitos orais. Atividades orais, como cerrar os dentes quando acordado, que geralmente são considerados como parte do BV, às vezes podem ser consideradas como hábitos orais e não estão necessariamente associados a consequências clínicas. Os participantes percebem o bruxismo de vigília e do sono como uma entidade única, fato que impossibilita o diagnóstico satisfatório por meio de autorrelato.

Para ambos os tipos de bruxismo a prevalência foi de 7,3\% (Winocur, et al.., 2019), contrastando com os dados de Murrieta, et al., (2014) de 47,5\%. Este último autor, não analisou cada tipo de bruxismo separadamente. Além disso, usou uma amostra bem menor quando comparada com os já citados. Nenhum dos dois justifica esse achado.

Dos dez artigos selecionados, nove aplicaram questionários com os adolescentes ou responsáveis. Para uma análise mais detalhada, Prado, et al., (2018), Sousa, et al., (2018) e Murrieta, et al., (2014) acrescentaram exame oral nos adolescentes. Esse é um fator importante, visto que é um método menos subjetivo no diagnóstico, se comparado somente com os questionários, que são fontes subjetivas e podem ser preenchidas erroneamente (Wetselaar et al., 2021; Prado, et al., 2018). Além disso, questionários preenchidos pelos pais também sofrem influência da convivência com os filhos nessa faixa etária, visto que, com o avanço da idade, os pais participam menos de alguns aspectos da vida do adolescente (Sousa, et al., 2018).

Carvalho, et al., (2015) demonstraram que as meninas tinham mais bruxismo do sono, do que os meninos, em torno de 53,8\% e na idade de 12 anos (31,1\%). Estes autores garantem que não é possível afirmar sobre a maior frequência de adolescentes com BS na idade citada, uma vez que a literatura é contraditória aos resultados encontrados. Sousa, et al., (2018) justificam seu achado da prevalência dos meninos no BS, ao relacionar o sexo masculino com um estado mais agitado nessa faixa etária da adolescência e por serem obrigados a reprimir suas emoções, podendo favorecer a ocorrência de movimentos involuntários. Esses autores também analisaram outras variáveis que Carvalho, et al., (2015) não observaram, como por exemplo, os hábitos dos adolescentes e o estado civil dos pais. Seus achados foram de 27,9\% para o sexo masculino e 18,9\% para o feminino, idade de 12 e 14 anos, com prevalência de 25,5\%.

A maioria dos autores relacionou o bruxismo ao sexo feminino, variando de aproximadamente $5 \%$ a $53,8 \%$ e a idade de 12 a 18 anos. Winocur, et al., (2019) ao estudarem os dois tipos de bruxismo, perceberam que em ambos, as meninas apresentaram maiores prevalências, com taxas de 24,1\% para o BS, e 32,1\% para o BV, em comparação com os meninos com $15,4 \%$ e $30,1 \%$, respectivamente. Com relação a idade, perceberam que adolescentes mais jovens sofriam mais de ambos os tipos de bruxismo do que os mais velhos. Mas ainda não há explicação para esse achado, visto que os estudos ainda se mostram muito contraditórios.

A comparação entre estudos de prevalência é dificultada por diferentes aspectos, para este estudo, os principais foram: países e populações estudadas, por conta de diferentes contextos sociais e culturais em que os adolescentes se inseriam, pois o ambiente também influencia no comportamento psicossocial e qualidade de vida; os métodos diagnósticos (questionários e/ou exame oral), visto que, análises através de registros polissonográficos e eletromiográficos se mostrariam mais eficazes nesse ponto, porém, acarretariam em altos custos, dificultando a abrangência das pesquisas. Além disso, o emprego de diferentes 
idades dentro da faixa etária da adolescência, emprego de idades específicas que podem sofrer influência do momento vivido pelo adolescente, não especificação do bruxismo (do sono ou vigília) e diversos fatores relacionados ao bruxismo.

Dentre os aspectos psicológicos mais citados nos artigos selecionados, observou-se a ansiedade e o estresse. A depressão foi investigada em apenas dois estudos. Selms, et al., (2013), perceberam a interferência da ansiedade e do humor depressivo, ao analisarem os dois tipos de bruxismo. Foi observada a associação positiva do BS e BV com estresse e sensação de tristeza. Pacientes depressivos são caracterizados por distúrbios no sistema neurotransmissor central. Nesse sentido, há necessidade de desmistificar a função dos neurotransmissores, principalmente dopamina, que são considerados fatores-chave na etiopatologia tanto dos transtornos de humor quanto do bruxismo.

Para Türkoğlu, et al., (2014), em bruxômanos noturnos, a proporção de pelo menos uma desordem psiquiátrica foi significativamente maior $(42,9 \%)$ do que para os casos controle $(17,1 \%)$, além da depressão e transtorno de ansiedade; a fobia social, o estresse pós-traumático e o transtorno do pânico, também fizeram parte das variáveis psiquiátricas. Apesar dos estudos que investigam a relação entre SB e gravidade dos sintomas psiquiátricos nas crianças e adolescentes serem limitados, os autores sugerem essa relação ao destacar a sensibilidade à ansiedade, independentemente da ansiedade ou gravidade dos sintomas depressivos. Isso sugere que a sensibilidade à ansiedade pode desempenhar um papel importante em possíveis casos de BS. Essas tendências também indicam a vulnerabilidade ao desenvolvimento de sintomas ou transtornos psiquiátricos nessa faixa etária.

Segundo Carvalho, et al., (2015), o BS aumentou 82\% a chance de alto impacto negativo na vida dos escolares. Também, apresentaram quase o dobro de chances de consequências na qualidade de vida quando comparado aos escolares sem BS; principalmente nas limitações funcionais e alterações no bem-estar social. Esses resultados podem ser explicados, pelas dores musculares provocadas pelo BS e pelo fato de a faixa etária estudada (11 aos 14 anos) apresentar melhor conhecimento sobre sua saúde e qualidade de vida, e habilidade de criar julgamentos sobre o seu bem-estar social.

Sobre a atividade física, o sexo masculino praticou mais atividade física comparado ao feminino. Navarro, et al., (2018) observaram a diferença entre os gêneros quanto aos dias, horas de exercício e horas de sedentarismo. Enquanto 35,4\% das meninas se exercitaram por 5 semanas ou mais; 53,9\% dos meninos se exercitaram durante o mesmo período. Cerca de 73,8\% dos escolares, relataram a participação em pelo menos atividades de intensidade leve. Todas as intensidades de AF, leve, média e vigorosa, tiveram médias maiores nos escolares do sexo masculino, segundo Shennar-Golan \& Walter (2018).

Essa tendência masculina pode ser explicada pela preocupação dos meninos com a comparação social com os de mesmo sexo, sendo as meninas mais preocupadas com sua aparência e influências da mídia; isso pode refletir na escolha dos níveis de atividade física e na prática ou não da mesma. Outro aspecto importante é a relação parental entre os meninos, que na maioria das vezes são estimulados desde cedo. Essa observação é reforçada quando os autores observam que, para os meninos, o relacionamento com os pais foi o único fator preditivo significativo de AF extenuante; para as meninas, o índide de bem-estar pessoal era o único preditor significativo (Shennar-Golan \& Walter, 2018).

Poucos estudos avaliaram a prevalência da prática de atividade física por idade. Apenas Freire, et al., (2014) e Santana, et al., (2021) se preocuparam com essa questão. Para Freire, et al., (2014), a prática regular de AF entre adolescentes apresentou associação estatisticamente significativa com: adolescentes mais jovens e ser do sexo masculino. Dados esses, que concordam com Bacil, et al., (2020) quando, observando sobre a maturidade na adolescência, destacou que a AF tende a diminuir com o avançar da idade, e uma das explicações para essa ocorrência pode ser a idade biológica, ou seja, o estado geral do corpo e o processo de envelhecimento. Em contrapartida, Santana, et al., (2021) descreveram que meninas mais novas se apresentaram mais inativas e sedentárias, cerca de $85,2 \%$ e $57,8 \%$ das meninas, respectivamente, versus $70,6 \%$ e $54,8 \%$ dos meninos. Nesse estudo, a faixa etária dos 11 aos 13 anos se apresentou mais inativa e sedentária, com taxas de 80,3\% e 59,2\%, 
respectivamente. Esses dados podem ser explicados pela supervisão parental, que pode diminuir os níveis de inatividade e sedentarismo dos filhos. Pais mais presentes criam uma relação de apoio e auxiliam na construção moral do adolescente.

Assim como no bruxismo, os aspectos psicológicos relacionados a atividade física mais citados foram a ansiedade, estresse, depressão ou tristeza, e também o bem-estar e a qualidade de vida. Para Ferreira, et al., (2020), existe uma correlação positiva entre atividade física no lazer e saúde mental. A chance de transtornos mentais comuns foi $16 \%$ maior no grupo que relatou ser inativo (0 minuto/semana). A prevalência de transtornos mentais comuns foi maior entre adolescentes que não praticaram esportes $(37,2 \%)$ comparado com os que praticavam $(25,9 \%)$. O mesmo para os que não participaram das aulas de educação física na escola (39,5\% vs. 29,6\%). Atividade física insuficiente (1-299 minutos/semana) não aumentou as chances de transtornos mentais comuns, mas não ofereceu nenhum benefício a mais para a saúde mental.

Praticar atividade física no lazer, independentemente da duração e frequência semanal, reduziu em $26 \%$ as chances de transtornos mentais comuns nessa população. A intensidade vigorosa de atividade física associou-se ao bem estar em estudantes de 13 a 18 anos. Esses dados confirmam o pressuposto de que praticar AF pode trazer benefícios psicológicos nessa fase da vida (Ferreira, et al., 2020; Shennar-Golan \& Walter, 2018).

Carrillo-Diaz, et al., (2021) analisou a mudança do estilo de vida dos adolescentes em um momento de restrição, em que desencadeou aumento dos quadros de sedentarismo, ansiedade, bruxismo clínico e bruxismo autorrelatado. Porém, nesse caso, não é possível estabelecer uma relação de causa e efeito direta entre a diminuição de atividade física, consequente do lockdown, a ansiedade desencadeada e o desenvolvimento do bruxismo entre os adolescentes; por estarem inseridos em condições atípicas e envolver outros aspectos, como por exemplo, o uso excessivo de telas digitais. Navarro, et al., (2018) não encontrou associação entre o nível de atividade física e presença, quantidade ou comportamento motor, como o bruxismo, em adolescentes.

Diante do exposto e das poucas pesquisas nesse âmbito, o bruxismo, sua relação com atividade física e aspectos psicológicos em adolescentes, ainda é um assunto para se aprofundar. Dos artigos selecionados, apenas um se aproximou do tema, investigando mudanças no estilo de vida (sedentarismo e aumento da ansiedade) e bruxismo. Os estudos mostram os benefícios envolvendo a prática de atividade física como um meio de preservar e melhorar a saúde, assim como a qualidade de vida do ser humano. A prática de atividade física é considerada uma forma benéfica de redução de estresse diário. Quando não eliminado, o estresse é capaz de promover grande aumento de tensão muscular (Freire, et al., 2014; Malinauskas \& Malinauskaiene, 2015; Navarro, et al., 2018).

A ansiedade, estresse e depressão foram relacionados ao bruxismo (Selms, et al., 2012; Wetselaar, et al., 2020; Türkoğlu, et al., 2013; Sousa, et al., 2018; Winocur, et al., 2019; Carrillo-Diaz, et al., 2021), impactando a qualidade de vida e bem-estar (Carvalho, et al., 2015), assim como a inatividade física (Parker, et al., 2016; Ferreira, et al., 2020; Freire, et al., 2014; Malinauskas \& Malinauskaiene, 2015; Carrillo-Diaz, et al., 2021; Shennar-Golan \& Walter, 2018). Mas nenhum dos autores citados ou selecionados, concordaram que todos esses aspectos estavam relacionados, ou se os aspectos psicológicos além de interferirem no bruxismo e na atividade física, interagem com esses dois, de maneira que, a prática de atividade física causasse algum impacto na vida psicológica do adolescente a ponto de este desenvolver ou não o comportamento motor do bruxismo.

\section{Considerações Finais}

Apesar de nem todos os artigos tratarem do BS e BV, a maioria, cujo o foco foram os dois tipos de bruxismo, descreveu o bruxismo do sono como mais prevalente na população de adolescentes. A idade foi um fator que diferiu entre os autores, além da falta de justificativa nesse âmbito, então, não foi possível determinar uma idade exata para uma maior 
prevalência, contudo, foi observado que os adolescentes mais novos foram mais propensos a apresentar esse comportamento (em torno de 12 a 14 anos). Em relação ao sexo, as meninas apresentaram mais frequência no desenvolvimento do bruxismo do sono e de vigília.

Sobre a atividade física, a maior parte descreveu a $\mathrm{AF}$ em: atividade, inatividade e sedentarismo. Os meninos praticaram mais atividade física que as meninas; e essas, tiveram maiores taxas de inatividade e sedentarismo. Dos dois autores que analisaram a idade, relataram informações contraditórias, o que torna inviável a conclusão.

Os fatores psicológicos mais envolvidos na pesquisa foram o estresse, ansiedade e depressão. A ansiedade foi mais frequente entre os achados, tanto no bruxismo, como na atividade física. Essas tendências indicam que os adolescentes são vulneráveis ao desenvolvimento de sintomas ou transtornos psicológicos. Assim, devem ser acompanhados pelos pais junto com professores e psicólogos, com o objetivo de intervir adequadamente.

Apesar do presente estudo sugerir uma relação entre bruxismo, atividade física e aspectos psicológicos, diante dos dados e como já foi visto, os fatores psicológicos se apresentam como fatores de risco para o desenvolvimento do bruxismo e esses mesmos fatores associam-se também, à atividade ou inatividade e/ou comportamento sedentário nos adolescentes. Mas não foi possível estabelecer uma relação direta nesse caso, visto que ainda não houveram estudos que comprovassem a veracidade desse assunto.

Diante disso, são necessários estudos aprofundados sobre o tema, visando toda população de adolescentes dentro da faixa etária da OMS e de preferência a longo prazo, para analisar os efeitos da prática de atividade física ou inatividade física nesse grupo. Além disso, métodos menos subjetivos de avaliação dos tipos de bruxismo, para garantir maior segurança no diagnóstico. Sobre os fatores psicológicos, a análise deve ser multiprofissional, a fim de abranger diversas esferas da vida do adolescente.

\section{Referências}

Alencar, L. B. B. de, Silva, I. L., Sousa, S. C. A. de, Araújo, V. F. de, Araújo, O. S. M. de, \& Moura, C. (2020). Associação do bruxismo diurno e noturno com a ansiedade: uma revisão integrativa. Research, Society and Development, 9(10). 10.33448/rsd-v9i10.9174

Alves, R. S. (2021). TeenPower: Plataforma mHealth para a prevenção da obesidade usando gamification para promover o exercício físico (Doctoral dissertation). Leiria, Portugal.

Antonio, A. G., Santos da Silva Pierro, V., \& Maia, L. C., et al. (2006). Bruxism in children: a warning sign for psychological problems. Journal of the Canadian Dental Association, 72(2).

Ayala, P. D., \& Llana, D. C. Z. (2019). El bruxismo en estudiantes de preuniversitario y su repercusión en el sistema estomatognático. Revista de Ciencias Médicas de Pinar del Río, 23(2), 269-277.

Bacil, E. D. A., Piola, T. S., Silva, M. P. D., Bozza, R., Fantineli, E., \& Campos, W. D., et al. (2020). Correlatos da atividade física em adolescentes de escolas públicas de Curitiba, Paraná. Revista Paulista de Pediatria, 38.

Carvalho, A. D. M. B., Lima, M. D. D. M. D., Silva, J. M. N. D., Neta, N. B. D., \& Moura, L. D. F. A. D. D. (2015). Bruxism and quality of life in schoolchildren aged 11 to 14. Ciência \& saúde coletiva, 20, 3385-3393. 10.11607/ofph.2687.

Carrillo Diaz, M., Ortega Martínez, A. R., Romero Maroto, M., \& González Olmo, M. J. (2021). Lockdown impact on lifestyle and its association with oral parafunctional habits and bruxism in a Spanish adolescent population. International Journal of Paediatric Dentistry. 10.1111/ipd.12843

De Carvalho Lírio, L. (2012). A construção histórica da adolescência. In Anais do Congresso Internacional da Faculdades EST 1, $1675-1688$.

Ferreira, V. R., Jardim, T. V., Póvoa, T. I. R., Viana, R. B., Sousa, A. L. L., \& Jardim, P. C. V. (2020). Inatividade física no lazer e na escola está associada à presença de transtornos mentais comuns na adolescência. Revista de Saúde Pública, 54. 10.11606/s1518-8787.2020054001888

Freire, R. S., Lélis, F. L. D. O., Fonseca, J. A. D., Nepomuceno, M. O., \& Silveira, M. F. (2014). Prática regular de atividade física: estudo de base populacional no Norte de Minas Gerais, Brasil. Revista Brasileira de Medicina do Esporte, 20, 345-349. https://doi.org/10.1590/1517-86922014200502062

Malinauskas, R., \& Malinauskaiene, V. (2015). Self-reported physical inactivity and health complaints: a cross-sectional study of Lithuanian adolescent schoolgirls. Cadernos de saúde publica, 31, 981-988. https://doi.org/10.1590/0102-311X00080614

Murrieta, J., Cielo, V., Morales, J., Sánchez, C., Linares, C., \& González, M.. (2014). Bruxism frequency and dental occlusion type in a group of Mexican adolescents. J. oral res. (Impresa), 211-217. 10.17126/joralres.2014.050 
Research, Society and Development, v. 11, n. 3, e22811326450, 2022

(CC BY 4.0) | ISSN 2525-3409 | DOI: http://dx.doi.org/10.33448/rsd-v11i3.26450

Navarro, G., Baradel, A. F., Baldini, L. C., Navarro, N., Franco-Micheloni, A. L., \& Pizzol, K. E. D. C.. (2018). Parafunctional habits and its association with the level of physical activity in adolescents. BrJP, 1, 46-50. 10.5935/2595-0118.20180010

Parker, A. G., Hetrick, S. E., Jorm, A. F., Mackinnon, A. J., McGorry, P. D., Yung, A. R., Purcell, R.. (2016). The effectiveness of simple psychological and physical activity interventions for high prevalence mental health problems in young people: a factorial randomised controlled trial. Journal of affective disorders, 196, 200-209. 10.1016/j.jad.2016.02.043

Prado, I. M., Abreu, L. G., Silveira, K. S., Auad, S. M., Paiva, S. M., Manfredini, D., \& Serra-Negra, J. M.. (2018). Study of associated factors with probable sleep bruxism among adolescents. Journal of Clinical Sleep Medicine, 14(8), 1369-1376. 10.5664/jcsm.7276

Santana, C. P., Nunes, H. A. S., Silva, A. N., \& Azeredo, C. M.. (2021). Associação entre supervisão parental e comportamento sedentário e de inatividade física em adolescentes brasileiros. Ciência \& Saúde Coletiva, 26, 569-580. 10.1590/1413-81232021262.07272019

Sousa, H. C. S., Lima, M. D. D. M. D., Dantas, N. B., Tobias, R. Q., Moura, M. S. D., \& Moura, L. D. F. A. D. D. (2018). Prevalência e fatores associados ao bruxismo do sono em adolescentes de Teresina, Piauí. Revista Brasileira de Epidemiologia, 21. 10.1590/1980-549720180002

Shennar-Golan, V., \& Walter, O. (2018). Physical activity intensity among adolescents and association with parent-adolescent relationship and wellbeing. American journal of men's health, 12(5), 1530-1540. 10.1177/1557988318768600

Türkoğlu, S., Akça, Ö. F., Türkoğlu, G., Akça, M. (2014). Psychiatric disorders and symptoms in children and adolescents with sleep bruxism. Sleep and Breathing, 18(3), 649-654. 10.1007/s11325-013-0928-y

Selms, M. K., Visscher, C. M., Naeije, M., Lobbezoo, F. (2013). Bruxism and associated factors among Dutch adolescents. Community dentistry and oral epidemiology, 41(4), 353-363. 10.1111/cdoe.12017

Wetselaar, P., Vermaire, E. J., Lobbezoo, F., \& Schuller, A. A. (2021). The prevalence of awake bruxism and sleep bruxism in the Dutch adolescent population. Journal of Oral Rehabilitation, 48(2), 143-149. 10.1111/joor.13117

Winocur, E., Messer, T., Eli, I., Emodi-Perlman, A., Kedem, R., Reiter, S., Friedman-Rubin, P. (2019). Awake and sleep bruxism among Israeli adolescents. Frontiers in neurology, 10, 443. 10.3389/fneur.2019.00443

World Health Organization. (2020). WHO guidelines on physical activity and sedentary behaviour: web annex: evidence profiles. 